\title{
Dietary Supplements and Sports Performance: Introduction and Vitamins
}

\author{
Melvin H. Williams, Ph.D., FACSM \\ Department of Exercise Science, Old Dominion University. Address correspondence to \\ profrunner@aol.com
}

Received November 9, 2004 /Accepted November 22, 2004/Published (online)

\begin{abstract}
Sports success is dependent primarily on genetic endowment in athletes with morphologic, psychologic, physiologic and metabolic traits specific to performance characteristics vital to their sport. Such genetically-endowed athletes must also receive optimal training to increase physical power, enhance mental strength, and provide a mechanical advantage. However, athletes often attempt to go beyond training and use substances and techniques, often referred to as ergogenics, in attempts to gain a competitive advantage. Pharmacological agents, such as anabolic steroids and amphetamines, have been used in the past, but such practices by athletes have led to the establishment of anti-doping legislation and effective testing protocols to help deter their use. Thus, many athletes have turned to various dietary strategies, including the use of various dietary supplements (sports supplements), which they presume to be effective, safe and legal. Journal of the International Society of Sports Nutrition. 1(2):1-6, 2004
\end{abstract}

Key Words: sport nutrition, dietary supplements, vitamins, sports performance

\section{INTRODUCTION}

Dietary supplements are used by athletes worldwide. In the United States, the Dietary Supplement Health and Education Act has defined dietary supplements as something added to the diet, mainly (1) vitamins, (2) minerals, (3) amino acids, (4) herbs or botanicals, and (5) metabolites/constituents/ extracts, or combination of any of these ingredients. In addition to actual food products targeted to athletes and physicallyactive individuals, numerous companies have marketed dietary supplements to athletes, often with the claim that sports performance may be enhanced. This is the first in a series of six articles to discuss the major classes of dietary supplements listed above. The major focus will be on efficacy of such dietary supplements to enhance exercise or sport performance, with brief coverage of safety, legality, and ethicality.

\section{VITAMINS: ERGOGENIC THEORY}

Vitamins function in the human body as metabolic regulators, influencing a number of physiological processes important to exercise or sport performance. For example, many of the B-complex vitamins are involved in processing carbohydrate and fats for energy production, an important consideration during exercise of varying intensity. Several B vitamins are also essential to help form hemoglobin in red blood cells, a major determinant of oxygen delivery to the muscles during aerobic endurance exercise.

Additionally, vitamins $\mathrm{C}$ and $\mathrm{E}$ function as antioxidants, important for preventing oxidative damage to cellular and subcellular structure and function during exercise training, theoretically optimizing preparation 
for competition. Complete details of vitamin functions and requirements are available in several recent treatises from the National Academy of Science ${ }^{1-4}$.

Vitamin deficiencies can certainly impair exercise performance. A daily intake of less than one-third of the RDA for several of the B vitamins $\left(B_{1}, B_{2}\right.$ and $\left.B_{6}\right)$ and vitamin $C$, even when other vitamins are supplemented in the diet, may lead to a significant decrease in $\mathrm{VO}_{2}$ max and the anaerobic threshold in less than four weeks ${ }^{5}$. However, most studies report that athletes who consume high-calorie diets that contain the RDA of all nutrients have few vitamin or mineral deficiencies ${ }^{6}$. Nevertheless, recent survey data indicate that vitamins are the most commonly used dietary supplements among various athletic groups ${ }^{7}$. Can vitamin supplementation above that provided by an adequate, healthy, balanced diet enhance sport or exercise performance?

\section{VITAMIN SUPPLEMENTS: EFFICACY}

Studies have been conducted to evaluate the ergogenic potential of virtually every individual vitamin, as well as clusters of vitamins and related substances, including the B-complex vitamins, multivitamin/mineral compounds, and antioxidants.

$B$ vitamins and Choline. As many of the $B$ vitamins are involved in the metabolism of carbohydrate, fat and protein, their ergogenic potential has been studied individually and in combination. In general, although a deficiency of the B vitamins may impair both aerobic and anaerobic exercise performance, supplementation has not been shown to enhance performance in well-nourished individuals. Niacin supplementation may influence fat metabolism, blocking the release of free fatty acids (FFA) from adipose tissue and increasing reliance on carbohydrate utilization, possibly leading to premature depletion of muscle glycogen. Some research has indicated that excess niacin supplementation may actually impair aerobic endurance performance ${ }^{8}$. Vitamins $\mathrm{B}_{1}, \mathrm{~B}_{6}$ and $\mathrm{B}_{12}$ are believed to affect the formation of serotonin, an important neurotransmitter involved in relaxation. Some research with large doses (60-200 times the RDA) of these vitamins has shown increases in fine motor control and performance in pistol shooting. Others have suggested that the beneficial effect was related to the role of these vitamins in promoting the development of neurotransmitters that induce relaxation ${ }^{9}$. Additional research is merited to evaluate these effects on performance in precision sports dependent on fine motor control. However, it should be noted that such doses could exceed the Tolerable Upper Intake Level (UL) for vitamin $\mathrm{B}_{6}$.

Choline, an amine, is found naturally in a variety of foods and its RDA is grouped with the $\mathrm{B}$ vitamins ${ }^{2}$. Choline is involved in the formation of acetylcholine, a neurotransmitter whose reduction in the nervous system may be theorized to be a contributing factor to the development of fatigue. Because plasma choline levels have been reported to be significantly reduced following marathon running, choline supplementation has been theorized to prevent fatigue. Research has shown that choline supplementation will increase blood choline levels at rest and during prolonged exercise, and some preliminary field and laboratory research has suggested increased plasma choline levels are associated with a significantly decreased time to run 20 miles. However, other wellcontrolled laboratory research has revealed that choline supplementation, although increasing plasma choline levels, exerted no effect on either brief, high-intensity anaerobic cycling tests or more prolonged aerobic exercise tasks ${ }^{10}$. For example, choline supplementation, although increasing plasma free choline in marathon runners, had no effect on predicted or actual marathon time ${ }^{11}$.

\section{MULTIVITAMIN/MINERALS}

The overall review of the literature supports the viewpoint that multivitamin/mineral supplements are unnecessary for athletes or 
other physically active individuals who are on a well-balanced diet with adequate calories. For example, several studies have provided multivitamin/mineral supplements over prolonged periods and reported no significant effects on both laboratory and sport-specific tests of physical performance ${ }^{12,13}$. In one of the most comprehensive studies, Telford and others ${ }^{14}$ evaluated the effect of long term (7-8 months) vitamin/mineral supplementation (100 to 5,000 times the RDA) on exercise performance of nationally ranked athletes in training at the Australian Institute of Sport. The athletes were tested on a variety of sportspecific tasks as well as common tests of strength, anaerobic power, and aerobic endurance. They reported no significant effect of the supplementation protocol on any measure of physical performance when compared to athletes whose vitamin and mineral RDA were met by normal dietary intake.

\section{ANTIOXIDANTS}

Antioxidant vitamins include vitamins $\mathrm{C}, \mathrm{E}$ and beta-carotene, while coenzyme $\mathrm{Q}_{10}$ $\left(\mathrm{CoQ}_{10}\right)$ is a lipid with vitamin characteristics. Antioxidant vitamins have been studied individually and collectively for their potential to enhance exercise performance or to prevent exercise-induced muscle tissue damage.

\section{Antioxidants and exercise performance.} Vitamin C supplementation has been shown to improve physical performance in vitamin C-deficient subjects, but several major reviews support the general conclusion that vitamin $\mathrm{C}$ supplementation does not enhance physical performance in well-nourished individuals ${ }^{15,16}$.

Vitamin E has been shown to enhance oxygen utilization during exercise at altitude ${ }^{17}$, but does not appear to be an effective ergogenic under sea level conditions ${ }^{18}$. A contemporary review indicated that although vitamin E supplementation may increase tissue or serum vitamin E concentration, most evidence suggests there is no discernable effect on training, performance, or rate of post-exercise recovery in either recreational or elite athletes ${ }^{19}$. CoQ 10 , also known as ubiquinone, is an antioxidant and may improve oxygen uptake in the mitochondria of the heart, and has been used therapeutically for the treatment of cardiovascular disease. Theoretically, improved oxygen usage in the heart and skeletal muscles could improve aerobic endurance performance. Only limited data are available, but these studies have shown that $\mathrm{CoQ}_{10}$ supplementation to healthy young or older subjects did not influence lipid peroxidation, heart rate, maximal oxygen uptake, anaerobic threshold, or cycling endurance performance ${ }^{20-23}$. One study reported that $\mathrm{CoQ}_{10}$ supplementation was associated with muscle tissue damage and actually impaired cycling performance compared to the placebo treatment ${ }^{24}$. Overall, a recent review ${ }^{25}$ concluded that there is limited evidence that dietary supplementation with antioxidants improves human performance.

\section{Antioxidants and muscle tissue damage.} Sen ${ }^{26}$ indicates that strenuous exercise may generate reactive oxygen species (ROS) to a level to overwhelm tissue antioxidant defense systems. The result is oxidative stress, and one possible outcome is oxidative damage to muscle tissues. Preventing muscle tissue damage during exercise training may help optimize the training effect and eventual competitive sports performance. Numerous studies have evaluated the potential of antioxidant vitamin supplementation to prevent exercise-induced muscle tissue damage, and several extensive reviews have evaluated the available literature. However, the viewpoints of the reviewers vary somewhat.

Several reviewers conclude that antioxidant vitamin supplementation does not appear to prevent exercise-induced muscle tissue damage. Goldfarb ${ }^{27}$ concluded that research findings, mostly conducted with vitamin $\mathrm{C}$, vitamin $\mathrm{E}$, and beta carotene, have indicated 
that clear evidence for their prophylactic effect on various types of muscle damage following exercise is lacking. Other reviews

${ }^{28}$ have indicated that although animal studies have shown some promising effects of antioxidant supplementation to lessen exercise-induced oxidative stress damage, studies with humans are less convincing.

Contrarily, Dekkers and others ${ }^{29}$ concluded that dietary supplementation with antioxidant vitamins has favorable effects on lipid peroxidation and exercise-induced muscle damage and recommend vitamin supplementation to individuals performing regular heavy exercise. Evans ${ }^{30}$ noted that several antioxidants, including vitamin $\mathrm{C}$ and especially vitamin $\mathrm{E}$, have been shown to decrease the exercise-induced increase in the rate of lipid peroxidation, which could help prevent muscle tissue damage. Other researchers ${ }^{31}$ are convinced that vitamin $\mathrm{E}$ contributes to preventing exercise-induced lipid peroxidation and possible muscle tissue damage, and recommend that athletes supplement with 100-200 milligrams of vitamin E daily to help prevent exerciseinduced oxidative damage. $\mathrm{Ji}^{32}$ indicates that the delicate balance between pro-oxidants and antioxidants suggests that supplementation of antioxidants may be desirable for physically active individuals under certain physiological conditions by providing a larger protective margin. In particular, $\mathrm{Ji}^{33}$ notes that the aging process lessens the exercise training-induced improvement in natural antioxidant enzymes and suggests exercise training in older athletes might be assisted with antioxidant supplementation in attempts to optimize antioxidant defense.

Sacheck and Blumberg ${ }^{34}$ concluded that the use of dietary antioxidants like vitamin $\mathrm{E}$ to reduce exercise-induced muscle injury have met with mixed success, which seems to be the prevailing viewpoint. All reviewers indicate more research is needed to address this issue and to provide guidelines for recommendations to athletes.

\section{VITAMIN SUPPLEMENTS: SAFETY, LEGALITY AND ETHICALITY}

Vitamin supplementation, particularly when limited to 100 percent of the RDA for each vitamin, is generally regarded as safe. However, excess amounts of several vitamins may contribute to serious health problems and tolerable upper limits (UL) have been established for many vitamins. For example, excessive amounts of vitamin A consumed by women who are pregnant may cause birth defects. Excessive amounts of niacin may contribute to liver damage. For complete details, consult the treatises by the National Academy of Science ${ }^{1-4}$.

The use of pure vitamin supplements by athletes is legal and ethical. However, some vitamin sports supplements marketed by unscrupulous entrepreneurs may contain banned substances. At the present time the dietary supplement industry is poorly regulated, and some preparations for athletes may be adulterated with banned substances, such as ephedrine. Athletes who consume vitamin supplements should purchase them only from reputable companies, such as those whose products that carry the USP (United States Pharmacopeia) certification on the label.

\section{VITAMIN SUPPLEMENTS AND SPORT $\backslash E X E R C I S E$ PERFORMANCE: SUMMARY}

In general, health professionals indicate that vitamin supplements are not necessary for the individual on a well-balanced diet, but they may be recommended for certain individuals, such as the elderly, vegans, and women of childbearing age. Moreover, some health professionals note that most people do not consume an optimal amount of vitamins by diet alone and indicate that it appears prudent for all adults to take vitamin supplements ${ }^{35-}$ ${ }^{36}$. In such cases, there is no need to take more than 100-150 percent of the RDA.

Obtaining adequate vitamins, including use of 
supplements, may also be prudent behavior for some athletes. Melinda Manore ${ }^{37,38}$ noted that athletes involved in heavy training may need more of several vitamins, such as thiamin, riboflavin and $\mathrm{B}_{6}$ because they are involved in energy production, but the amount needed is only about twice the RDA and that may be easily obtained through increased food intake associated with heavy training. However, in a recent scientific roundtable exchange ${ }^{39}$, several sport nutrition experts indicated that some athletes may be at risk for a vitamin deficiency, such as those in weightcontrol sports and those who for one reason or another do not eat a well-balanced diet. Others note that the prudent use of antioxidant supplementation can provide insurance against a suboptimal diet and/or the elevated demands of intense physical activity, and thus may be recommended to limit the effects of oxidative stress in individuals performing regular, heavy exercise.

\section{REFERENCES:}

1. National Academy of Sciences. Dietary Reference Intakes for Vitamin A, Vitamin K, Arsenic, Boron, Chromium, Copper, Iodine, Iron, Manganese, Molybdenum, Nickel, Silicon, Vanadium, and Zinc. Washington, DC: National Academy Press, 2002.

2. National Academy of Sciences. Dietary Reference Intakes for Thiamin, Riboflavin, Niacin, Vitamin B6, Folate, Vitamin B12, Pantothenic Acid, Biotin, and Choline. Washington, DC: National Academy Press. 2000A.

3. National Academy of Sciences. Dietary Reference Intakes for Vitamin C, Vitamin E, Selenium and Carotenoids. Washington, DC: National Academy Press. 2000B.

4. National Academy of Sciences. Dietary Reference Intakes for Calcium, Phosphorus, Magnesium, Vitamin D, and Fluoride. Washington, DC: National Academy Press. 1999.

5. van der Beek, E. Vitamin supplementation and physical exercise performance. Journal of Sport Sciences 1991;92:77-79,

6. Armstrong, L.; Maresh, C. Vitamin and mineral supplements as nutritional aids to exercise performance and health. Nutrition Reviews1996;54 Suppl S148-158.

7. Jacobson, B., et al. Nutrition practices and knowledge of college varsity athletes: A follow-up. Journal of Strength and Conditioning Research 2001;15:63-8,

8. Bulow, J. Lipid metabolism and utilization. In Poortmans, J. Principles of Exercise Biochemistry. Basel, Switzerland: Karger, 1993.

9. Bonke, D. Influence of vitamin B1, B6 and B12 on the control of fine motoric movements. Bibliotheca Nutritio et Dieta. 1986;38: 104-9.

10. Williams, M. H. Nutrition for Health, Fitness \& Sports. Boston: McGraw-Hill, 2004.

11. Buchman, A., et al. 2000. The effect of lecithin supplementation on plasma choline concentrations during a marathon. Journal of the American College of Nutrition 2000;19:768-70.

12. Singh, A., et al. Chronic multivitamin-mineral supplementation does not enhance physical performance. Medicine and Science in Sports and Exercise 1992;24:726-32,

13. Weight, L., et al. Vitamin and mineral supplementation: Effect on the running performance of trained athletes. American Journal of Clinical Nutrition 1998;47:192-95,.

14. Telford, R., et al. The effect of 7 to 8 months of vitamin/mineral supplementation on athletic performance. International Journal of Sport Nutrition 1992;2:135-53,

15. Gerster, H. Review: The role of vitamin C in athletic performance. Journal of the American College of Nutrition 1989;8:63643.

16. Williams, M. H. Vitamin supplementation and athletic performance. International Journal for Vitamin and Nutrition Research Supplement 1989;30:161-91.

17. Simon-Schnass, I.; Pabst, H. Influence of vitamin E on physical performance. International Journal of Vitamin and Nutrition Research 1988;58:49-54.

18. Rokitski, L., et al. $\alpha$-tocopherol supplementation in racing cyclists during extreme endurance training. International Journal of Sport Nutrition 1994;4:253-64,.

19. Tiidus, P., and Houston, M. Vitamin E status and response to exercise training. Sports Medicine 1995;20:12-23.

20. Bonetti, A., et al. Effect of ubidecarenone oral treatment on aerobic power in middle-aged trained subjects. Journal of Sports Medicine and Physical Fitness 2000;40:51-7.

21. Braun, B., et al. The effect of coenzyme Q10 supplementation on exercise performance, $\mathrm{VO}_{2}$ max, and lipid peroxidation in trained cyclists. International Journal of Sport Nutrition 1991;1:353-65.

22. Laaksonen, R., et al. Ubiquinone supplementation and exercise capacity in trained young and older men. European Journal of Applied Physiology 1995;72:95-100.

23. Snider, I., et al. Effects of coenzyme athletic performance system as an ergogenic aid on endurance performance to exhaustion. International Journal of Sport Nutrition 1992;2:272-86.

24. Malm, C., et al. Supplementation with ubiquinone-10 causes cellular damage during intense exercise. Acta Physiologica Scandinavica 1996;157:511-12.

25. Powers, S; Hamilton, K. Antioxidants and exercise. Clinics in Sports Medicine 1999;18:525-36,

26. Sen, C. Antioxidants in exercise nutrition. Sports Medicine 2001;31:891-908.

27. Goldfarb, A. Nutritional antioxidants as therapeutic and preventive modalities in exercise-induced muscle damage. Canadian 
Journal of Applied Physiology 1999;24:248-66.

28. Adams, A.; Best, T. The role of antioxidants in exercise and disease prevention. Physician and Sports Medicine 2002;30(6):3744.

29. Dekkers J., et al. The role of antioxidant vitamins and enzymes in the prevention of exercise-induced muscled damage. Sports Medicine 1996;21:213-38.

30. Evans, W. Vitamin E, vitamin C, and exercise. American Journal of Clinical Nutrition 2000;72:647S-52S.

31. Takanami, Y., et al. Vitamin E supplementation and endurance exercise: Are there benefits? Sports Medicine 2000; $29: 73-83$.

32. Ji, L. Antioxidants and oxidative stress in exercise. Proceedings of the Society for Experimental Biology and Medicine 1999;222:283-92.

33. Ji, L. Exercise-induced modulation of antioxidant defense. Annals of the New York Academy of Sciences 2002;959: 82-92.

34. Sacheck, J.; Blumberg, J. Role of vitamin E and oxidative stress in exercise. Nutrition 2001;17:809-14.

35. Fairfield, K.; Fletcher, R. Vitamins for chronic disease prevention in adults: Scientific review. JAMA 2002;287: 3116-26.

36. Fletcher, R.; Fairfield, K. Vitamins for chronic disease prevention in adults: Clinical applications. JAMA 2002;287: 3127-9.

37. Manore, M. Vitamins and minerals: Part I. How much do I need? ACSM's Health \& Fitness Journal 2001A; 5(3):33-35.

38. Manore, M. Vitamins and minerals: Part II. Who needs to supplement? ACSM's Health \& Fitness Journal 2001; 5(4):30-34.

39. Benardot, D., et al. Can vitamin supplements improve sport performance? Sports Science Exchange Roundtable 2001;12(3):1-4. 